\title{
Magnetism of FePt Nanoclusters in Polyimide
}

\author{
Mircea Chipara, ${ }^{1}$ Tom George, ${ }^{2}$ Yingfan $X u_{,}{ }^{2}$ Ralph Skomski, ${ }^{2}$ Lanping Yue, ${ }^{2}$ \\ Nasar Ali, ${ }^{3}$ and David J. Sellmyer ${ }^{2}$ \\ ${ }^{1}$ Department of Physics and Geology, The University of Texas Pan American, 1201 W. University Drive, Edinburg, TX 78504, USA \\ ${ }^{2}$ Department of Physics and Astronomy, University of Nebraska, Lincoln, NE 68588, USA \\ ${ }^{3}$ Department of Electrical Engineering, Meliksah University, Talas, 38289 Kayseri, Turkey
}

Correspondence should be addressed to Mircea Chipara; mchipara@utpa.edu

Received 4 December 2014; Revised 29 March 2015; Accepted 31 March 2015

Academic Editor: Mohamed Bououdina

Copyright (C) 2015 Mircea Chipara et al. This is an open access article distributed under the Creative Commons Attribution License, which permits unrestricted use, distribution, and reproduction in any medium, provided the original work is properly cited.

\begin{abstract}
FePt nanoclusters have been implanted onto polyimide films and subjected to thermal annealing in order to obtain a special magnetic phase $\left(\mathrm{Ll}_{0}\right)$ dispersed within the polymer. SQUID measurements quantified the magnetic features of the as-prepared and annealed hybrid films. As-implanted FePt nanoparticles in polyimide films exhibited a blocking temperature of $70 \pm 5 \mathrm{~K}$. Thermal annealing in zero and $10 \mathrm{kOe}$ applied magnetic field increased the magnetic anisotropy and coercivity of the samples. Wide Angle X-Ray Scattering confirmed the presence of FePt and $\mathrm{L}_{0}$ phase. All samples (as deposited and annealed) exhibited electron spin resonance spectra consisting of two overlapping lines. The broad line was a ferromagnetic resonance originating from FePt nanoparticles. Its angular dependence indicated the magnetic anisotropy of FePt nanoparticles. SEM micrographs suggest a negligible coalescence of FePt nanoparticles, supporting that the enhancement of the magnetic properties is a consequence of the improvement of the $\mathrm{Ll}_{0}$ structure. The narrow ESR line was assigned to nonmagnetic (paramagnetic) impurities within the samples consistent with graphite-like structures generated by the local degradation of the polymer during implantation and annealing. Raman spectroscopy confirmed the formation of graphitic structures in annealed KHN and in KHN-FePt.
\end{abstract}

\section{Introduction}

Synthesis of magnetic (metal) clusters at submicron scale by gas aggregation is based on target atoms evaporating/sputtering into a cooled inert gas flow at relatively high pressure [1]. Collisions cascade between metallic atoms and carrier gas results in the nucleation of supersaturated metal vapors into clusters. This method produces high purity clusters with diameters below $10 \mathrm{~nm}$ and deposition rates of the order of $1 \AA / s[1,2]$. FePt clusters with an average diameter of about $5 \mathrm{~nm}$ and a narrow distribution of diameters (characterized by a ratio between the standard deviation of $\mathrm{FePt}$ diameters, $\sigma$, and the diameter of the cluster, $d$ equal to or less than 0.1 [2]) have been obtained by the system built at the University of Nebraska, Lincoln.

The lattice symmetry of FePt is face cubic centered (fcc). The $\mathrm{Ll}_{0}$ phase has an "ordered" slightly distorted fcc structure (actually is a face centered tetrahedral lattice), as beyond the lattice ordered structure, the base atoms ( $\mathrm{Fe}$ and $\mathrm{Pt})$ are orderly distributed (actually one atom occupies two opposite faces of the crystalline lattice and the other atoms occupy the remaining sites) [3]. Hence, $\mathrm{Ll}_{0}$ has two degrees of symmetry, a lattice symmetry, identified as face centered tetrahedral ( $\mathrm{fct}$ ) lattice, and a base atom (distribution) symmetry consisting of alternating planes of $\mathrm{Fe}$ and $\mathrm{Pt}$ atoms along the $c$ axis [4]. The deviation of the $\mathrm{Ll}_{0}$ phase from the fcc structure is very small with the " $a$ " ranging between 3.85 and $3.87 \mathrm{~A}$ and " $c$ " ranging between 3.71 and $3.73 \mathrm{~A}$ (depending on synthesis path and annealing temperature [4]), where a perfect fcc structure implies $c=a$. The degree of "atom base order" in FePt is quantified by the ordering parameter $S[5]$ :

$$
S=c \sqrt{\frac{I_{001}}{I_{002}}},
$$

where $c$ is a constant (equal to about 0.49 according to [6] and to 0.85 according to [7]), $I_{001}$ is the amplitude of the 001 reflection observed at about $21.8^{\circ}$, and $I_{002}$ is the amplitude of 
the 002 reflection noticed at about $47.9^{\circ}$. A perfect $\mathrm{L1}_{0}$ phase corresponds to $S=1$. The formation of the $\mathrm{L1}_{0}$ phase in FePt:C systems is affected by the carbon content, showing a maximum ordering parameter for about $15 \%$ vol carbon [8]. Upon annealing, the diffraction line located at about $49^{\circ}$ is narrowed and eventually the splitting between the (220) and (202) reflections becomes visible. The out of plane coercivity was reported to be maximum for $15 \%$ vol. carbon in FePt granular systems, reflecting the strong connection between the structure of the FePt nanoparticles and their magnetic features [8]. For FePt nanoparticles obtained by chemical reduction, very good $\mathrm{Ll}_{0}$ phases have been obtained after thermal annealing at temperatures higher than $650^{\circ} \mathrm{C}$ (with a maximum of coercivity and grain size at $750^{\circ} \mathrm{C}$ and additional contributions originating from the annealing time) [7]. Recent studies indicated that the order parameter is decreasing as the heating rate is increased [9], reaching a saturation value at high cooling rates.

This unusual double order (lattice and atom base) of the $\mathrm{L}_{0}$ phase is responsible for its outstanding magnetic properties making $\mathrm{L}_{0}$ phase one of the most promising candidates for future ultrahigh density perpendicular magnetic media due to its high magnetocrystalline anisotropy $\left(6.6 \mathrm{Merg} / \mathrm{cm}^{3}\right)$, which is capable of suppressing thermal agitation in spins at the nanometer scale [10-13]. In the case of FePt nanoparticles obtained by chemical reduction, the assynthesized samples have been reported to show superparamagnetic features at room temperature [4]. The coercivity of these FePt nanoparticles increases as the annealing temperature is increased reaching a maximum of about $22.7 \mathrm{kOe}$, for an annealing temperature of $800^{\circ} \mathrm{C}$ [4]. The coercivity of FePt:C has a strong dependence on the carbon content and on the orientation of the sample. The angular dependence of the coercivity was found to exhibit a maximum for films oriented at about 60 to $75^{\circ}$ (with respect to the normal to the film) [8]. The coercivity at these angles is larger than the coercivity in the parallel or perpendicular configurations. Nevertheless, the maximum value of coercivity decreases as the $\mathrm{C}$ concentration increases, consistent with a gradual transition from a domain wall motion to a rotation mode motion [8]. The coercivity of the FePt:C was reported to be larger for the normal configuration (film perpendicular to the magnetic field) than for the parallel one [8].

The magnetization at saturation of FePt nanoparticles obtained by chemical reduction was reported to increase up to about $1100 \mathrm{emu} / \mathrm{cm}^{3}$ for an annealing temperature of about $600^{\circ} \mathrm{C}$. Higher annealing temperatures resulted in a decrease of the magnetization at saturation [4]. However, no significant deviations of the magnetization squareness of the hysteresis loop were reported for FePt:C films with less than $20 \%$ (vol) carbon (as-deposited or thermally annealed at low temperatures, below $350^{\circ} \mathrm{C}$ ) [8]. The raise of the thermal annealing temperature (up to $350^{\circ} \mathrm{C}$ ) increased the coercivity of the FePt:C samples, with the out of the plane coercivity larger than the in plane coercivity [8]. The hysteresis loops recorded in the perpendicular configuration have smaller coercive fields and magnetization at saturation than the same hysteresis loops recorded in the parallel configuration.
Actually, for the perpendicular configuration, the hysteresis loops are not saturated even in magnetic fields of about 10,000 Oe [14].

Flexible high density recording media may be obtained by dispersing such nanoparticles within polymeric matrices [11] via implantation or chemical synthesis. Magnetically decoupling the nanoparticles by segregation in a nonmagnetic matrix reduces media noise and allows for higher areal recording densities. Particular effort has been concentrated on the investigations of FePt nanoparticles dispersed within $\mathrm{C}$ (so called FePt:C system [12]). The magnetic properties of the analogous system represented by CoPt nanoparticles dispersed within polymethyl methacrylate have been reported [15], while only few articles have been published on the $\mathrm{Ll}_{0}$ phase of FePt nanoparticles in polymeric materials (search engine Web of Knowledge). Among these reports, there is a study on the synthesis of polymethyl methacrylate, FePt nanocomposites, which demonstrated magnetic properties at very low temperatures without any structural analysis (and hence no detail about the $\mathrm{Ll}_{0}$ phase) [16]. An inciting study on the three-dimensional organization of FePt within polymer composites obtained by chemical synthesis was reported in $[17,18]$. However, the authors did not focus on the crystalline structure of FePt and did not investigate the $\mathrm{Ll}_{0}$ phase presence and characteristics. The encapsulation of FePt nanoparticles within nanofibers of poly( $\varepsilon$ caprolactone $)$ and the superparamagnetic nature of the hysteresis loop at room temperature have been reported, without any detail about the nature of the crystalline phase [19-21]. The orientation of FePt nanoparticles under the effect of an external magnetic field during the polymerization has been recently reported [22]. The study investigated by Mossbauer spectroscopy the $\mathrm{Ll}_{0}$ phase and the orientation of nanoparticles. The authors did not find any study on implanted FePt nanoparticles or clusters into polymeric matrices.

\section{Materials and Methods}

FePt nanoclusters were obtained by gas aggregation technique $[1,2]$. Nanocomposites of FePt in polyimide (labelled as $\mathrm{KHN}-\mathrm{FePt}$ ) have been obtained by implanting nanoclusters of FePt within free standing polyimide (Kapton HN; DuPont) films, with a thickness of about 0.0254 millimeters.

The polymeric substrate, polyimide Kapton HN (KHN), is a semicrystalline polymer, which decomposes in air at about $525^{\circ} \mathrm{C}$ (before melting). The thermal annealing of $\mathrm{KHN}-\mathrm{FePt}$ has been performed at various temperatures below the decomposition temperature of KHN. The last step of the annealing process was a fast cooling that prevented the eventual crystallization of KHN. Hence, the WAXS lines of the polymeric matrix are expected to be broad and weak. Even more, upon implantation with FePt nanoparticles, the degree of crystallinity of the polymeric matrix is expected to decrease, in agreement with experimental data on ion bombardment effects on this polymer [23].

To enhance the quality of the $\mathrm{L}_{0}$ phase, the as-obtained films were annealed by rapid thermal annealing (RTA, at a heating rate of $100^{\circ} \mathrm{C} / \mathrm{s}$ in inert atmosphere) or 

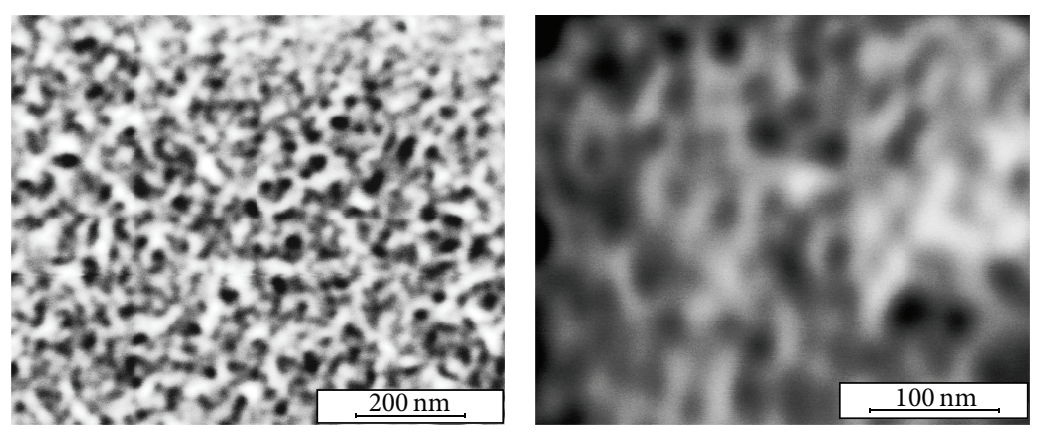

(a)

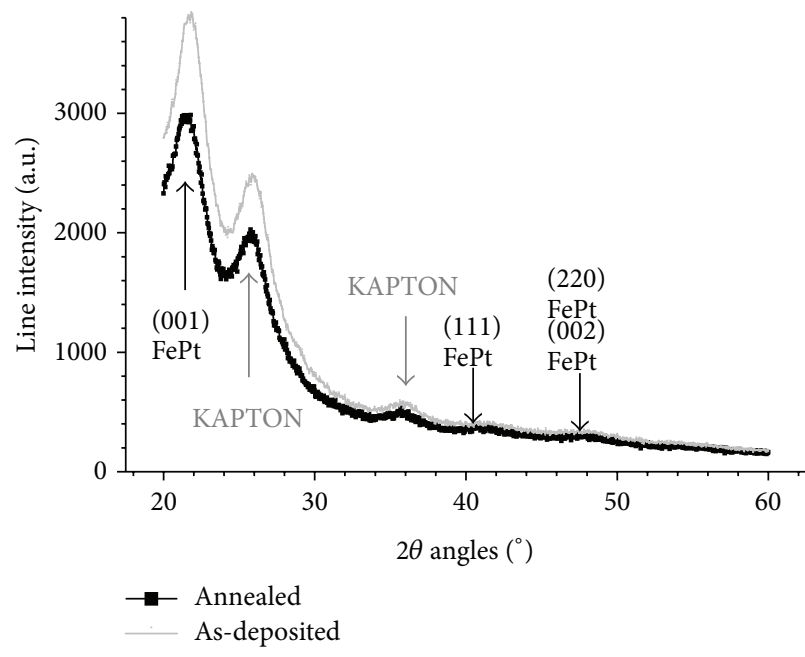

(b)

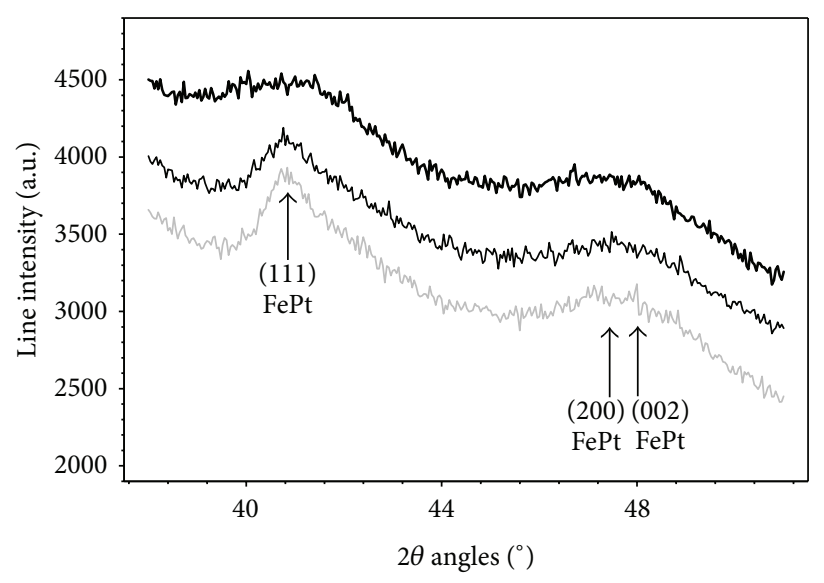

(c)

Figure 1: (a) SEM photographs of KHN-FePt. WAXS of KHN-FePt. (b) Not annealed KHN-FePt (thick black line) and KHN-FePt annealed at $400^{\circ} \mathrm{C}$ for 10 minutes by RTA (thin gray line). (c)WAXS of as-deposited $\mathrm{KHN}-\mathrm{FePt}$ (thick black line), of $\mathrm{KHN}-\mathrm{FePt}$ annealed at $460^{\circ} \mathrm{C}$ for $1 \mathrm{~h}$ in $H$ (parallel) $=10 \mathrm{kOe}$ (thin black line), and of $\mathrm{KHN}-\mathrm{FePt}$ annealed at $460^{\circ} \mathrm{C}$, for $1 \mathrm{~h}$, in $H$ (perpendicular) $=10 \mathrm{kOe}$ (thick gray line).

within a Vibrating Sample Magnetometer (VSM) at various temperatures ranging between $400^{\circ} \mathrm{C}$ and $500^{\circ} \mathrm{C}$. In order to improve the orientation of magnetic nanoclusters, some of the samples have been annealed in external magnetic field (by using the VSM) of $10 \mathrm{kOe}$ for one hour. The annealing has been done in two configurations, the parallel one $(\|)$ with the external magnetic field along the plane of the film, and the perpendicular one $(\perp)$, with the external magnetic field perpendicular to the plane of the film.

The magnetic features (magnetization, hysteresis loops, and magnetic anisotropy) of the as-deposited and annealed films have been acquired by SQUID magnetometry and ferromagnetic resonance (FMR), in different configurations. Magnetic properties of $\mathrm{KHN}-\mathrm{FePt}$ at various temperatures in the range $10 \mathrm{~K}-700 \mathrm{~K}$ have been obtained and analyzed. The structure of Fe-Pt nanoparticles dispersed in polyimide was confirmed by Wide Angle X-Ray Scattering (WAXS). Additional Raman spectroscopy measurements were performed by using a Bruker Senterra confocal Raman microscope. Scanning Electron Microscopy photos of the nanoclusters within the polymer have been obtained by a FEI NOVA NANO SEM 450.

\section{Results and Discussion}

The SEM photographs of polyimide bombarded with FePt nanoclusters and annealed at $400^{\circ} \mathrm{C}$ are shown in Figure 1(a). The photos show that the coalescence of the FePt nanoparticles is negligible and suggest that the FePt nanoparticles are eventually embedded within a layer which resulted from the local modification of the polymeric matrix. ESR and Raman spectroscopy provided further details about the interface between KHN and FePt nanoclusters. WAXS spectra of the as-deposited KHN-FePt sample (see Figures 1(b) and 1(c)) show the lines assigned to (001), (110), (020), and (002) reflections in FePt $[3,8,24,25]$. For FePt nanoparticles obtained by chemical reductions the lines assigned to (001) and (110) reflections have been reported as absent [4], while in the case of $\mathrm{FePt}$ nanoclusters these lines are well resolved. WAXS spectra of FePt nanoparticles synthesized through the reduction of iron acetylacetonate and platinum acetylacetonate by 1,2-hexadecanediol in dioctyl ether [26] revealed a very broad line located at about $49^{\circ}$, which dominates the spectrum of FePt. As seen from Figure 1(b), this line is weak and broad in the case of as-obtained FePt nanoclusters. The lines at about $25.9^{\circ}$ and $36^{\circ}$ displayed in Figure 1(b) have been 


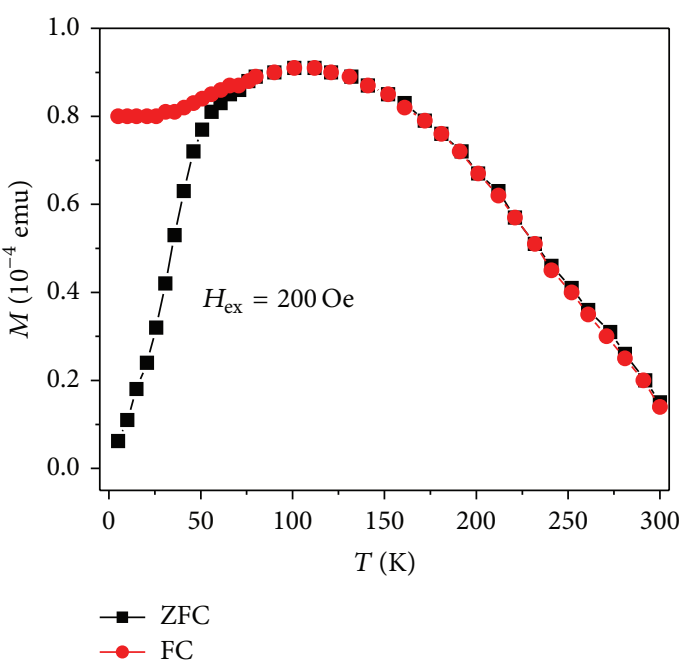

(a)

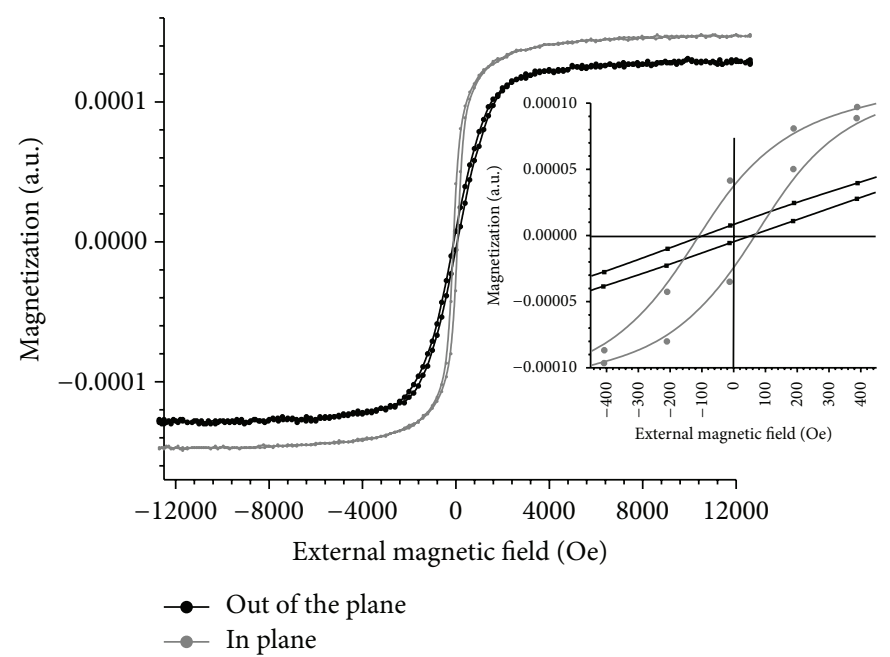

(b)

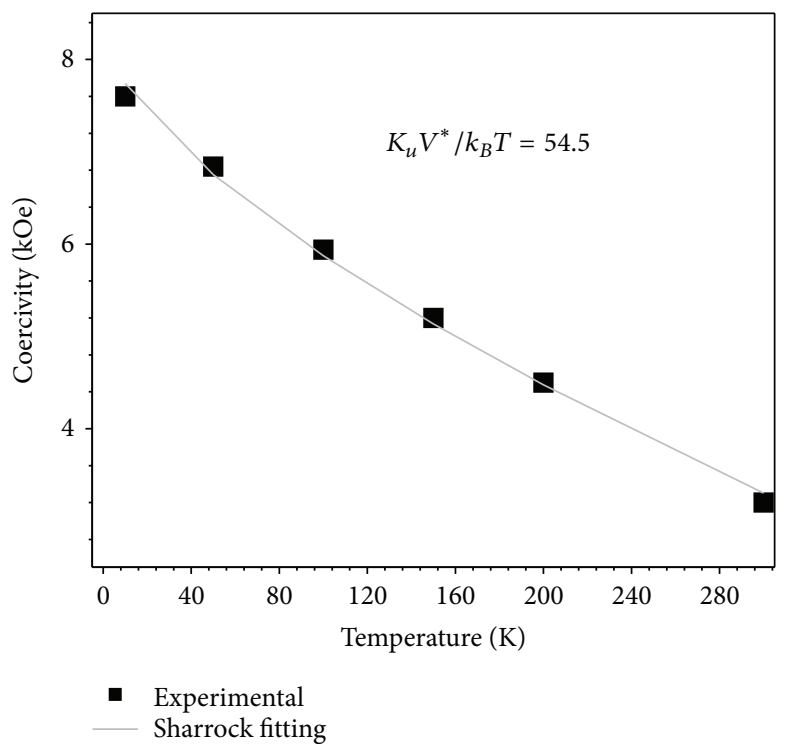

(c)

Figure 2: Magnetic data for as-deposited KHN-FePt (a). FC and ZFC temperature dependence of the magnetization (b). Hysteresis loops for the in plane and out of the plane configurations. (c). Temperature dependence of coercivity for annealed KHN-FePt.

assigned to $\mathrm{KHN}[23]$; the $25.9^{\circ}$ reflection corresponds to the strongest line reported in KHN [23].

The inspection of Figures 1(b) and 1(c) indicates (001) reflection and a broad and weak (002) reflection, showing that the as-deposited nanoclusters have an important fraction of $\mathrm{Ll}_{0}$ phase. The annealing at $410^{\circ} \mathrm{C}$ for 10 minutes does not change significantly the WAXS spectrum. Figure 1(c) includes the spectra of the as-deposited KHN-FePt film and of KHN-FePt annealed at $460^{\circ} \mathrm{C}$ in external magnetic fields of 10,000 Oe oriented parallel and, respectively, perpendicular to the direction of KHN-FePt film. It is noticed that WAXS cannot reveal major modifications upon thermal annealing.

The as-deposited FePt nanoclusters within polyimide have superparamagnetic features, as can be inferred from Figure 2(a), which depicts the temperature dependence of the magnetization for the so-called field cooled (FC) and zero field cooled (ZFC) branches. For the not annealed sample, the temperature dependence of the magnetization of FC and ZFC branches is consistent with a blocking temperature, $T_{B}$ of about $70 \pm 5 \mathrm{~K}$. The $\mathrm{FC}$ magnetization has been recorded in an applied magnetic field of 200 Oe. For magnetic nanoparticles, the blocking temperature depends on the size of the nanoparticles via their volume $V$ according to the following equation $[11,27,28]$ :

$$
T_{B}=\frac{K_{1} V}{k_{B} \ln \left(t_{m} / t_{0}\right)},
$$

where $K_{1}$ is the axial magnetic anisotropy, $k_{B}$ is Boltzmann constant, $t_{0}$ is a time characteristic to the material, and $t_{m}$ 
is the time scale of the experiment, about $100 \mathrm{~s}$ for SQUID measurements. Consequently, for this experiment, $\ln \left(t_{m} / t_{0}\right)$ is about 25. Previous microscopy data indicated that the size of FePt nanoclusters is about $5 \mathrm{~nm}$ [29]. This implies uniaxial magnetocrystalline anisotropies of the order of (3.7 \pm $0.3) \mathrm{Merg} / \mathrm{cm}^{3}$.

Figure 2(b) shows the hysteresis loop at room temperature of the as-obtained KHN film loaded with FePt nanoclusters for the parallel and perpendicular configurations. It is noticed that the coercivity of this magnetic nanomaterial at room temperature is small but not zero. Actually, the coercivities for the parallel and perpendicular configurations are almost identical suggesting a random distribution of anisotropy fields although the remnant magnetization is larger for the perpendicular configuration.

The temperature dependence of the coercivity, $H_{\mathrm{CR}}$, for the sample annealed in the VSM (magnetic field perpendicular to the plane of the sample) shown in Figure 2(c), is well described by Sharrock equation [30, 31]:

$$
H_{\mathrm{CR}}=H_{0}\left[1-\left(\frac{k_{B} T}{K_{1} V} \ln \left(\frac{t_{m}}{t_{0}}\right)\right)^{n}\right],
$$

where $H_{\mathrm{CR}}$ is the remnant coercive field, $n$ is either $1 / 2$ (for the case of particles aligned along the direction of magnetic field subjected to coherent rotations of magnetization during the nucleation [32]) or smaller (another frequently used value is $n=2 / 3$ [31]) and it is related to the orientation of the anisotropy axis. $H_{0}$ represents the intrinsic remnant coercivity [33]. The fit shown in Figure 2(c) was obtained assuming that $n=2 / 3$.

In order to enhance the coercivity of this sample and to move the blocking temperature towards higher temperature, two annealing techniques have been used. The first annealing procedure was performed in a rapid thermal annealing system (RTA), with temperature ramp rate of $100^{\circ} \mathrm{C} / \mathrm{s}$, up to $450^{\circ} \mathrm{C}$, an isothermal annealing for 10 minutes at this temperature followed by a rapid cooling. This annealing has been performed with no external magnetic field. The fast heating and cooling of the KHN-FePt sample did not allow the recrystallization of the polymeric film. Figure 3 collects the hysteresis loops of KHN-FePt samples annealed under these conditions. The in plane and out of plane hysteresis loops have been recorded at two temperatures, room temperature and $10 \mathrm{~K}$. It is noticed that the annealing at $450^{\circ} \mathrm{C}$ increased significantly the coercivity at room temperature up to $1700 \mathrm{Oe}$ in the perpendicular configuration, at room temperature. Hence, $\mathrm{KHN}-\mathrm{FePt}$ samples annealed at $450^{\circ} \mathrm{C}$ or more exhibit a ferromagnetic behavior with a large coercive field. As expected, the decrease of the temperature results in a substantial enhancement of the coercivity. Figure 3 and Table 1 suggest an in plane easyaxis texture probably via the preferential alignment of the easy axis of magnetization (shape anisotropy is almost zero as these magnetic nanoparticles are almost spherical). This may explain why the coercivity of annealed samples is not the same for the parallel and perpendicular configurations. Experimental data indicates that the magnetic coercivity is larger in the parallel configuration (external magnetic field
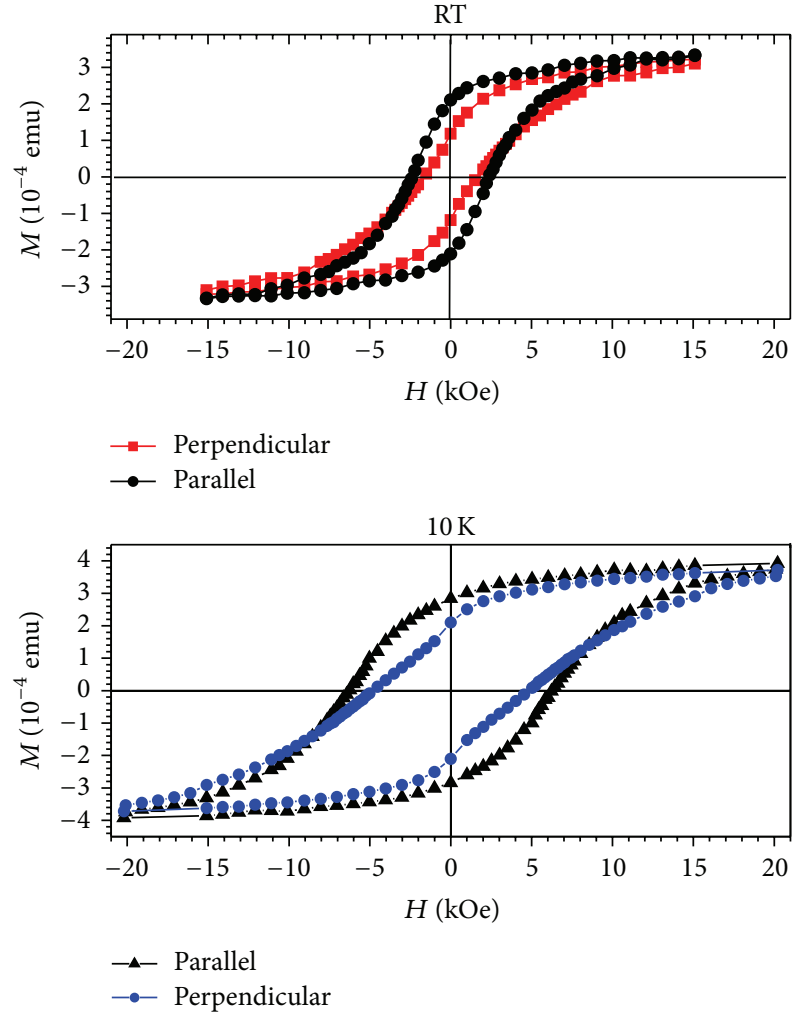

FIgURE 3: Hysteresis loops of KHN-FePt samples after an RTA annealing at $450^{\circ} \mathrm{C}$ for 10 minutes. The hysteresis loops have been recorded in parallel and perpendicular configurations, at room temperature and $10 \mathrm{~K}$.

parallel to the plane of the film) than in the perpendicular configuration, in contrast with most magnetic studies on FePt nanoparticles [8]. Nevertheless, it is important to notice that, in FePt films, the highest coercivity does not occur neither in the parallel nor in the perpendicular configuration [8]. This suggests that the rotation mode of the magnetization relaxation is somehow hindered by the polymeric matrix and the dominating magnetic relaxation is now controlled by wall motion [8].

Tentatively, a thermal annealing has been performed in the VSM with applied external magnetic field. The thermal annealing was done at $460^{\circ} \mathrm{C}$ for 60 minutes in two configurations: one with the external magnetic field perpendicular to the plane of the KHN-FePt film (perpendicular annealing configuration, see Figure 4(a)) and the other with the external magnetic field within the plane of the film (parallel annealing configuration, see Figure 4(b)). Figure 4 collects the hysteresis loops for the films annealed within the VSM in both configurations at $10 \mathrm{~K}$ and $300 \mathrm{~K}$. From Figure 4 it is noticed that the coercive field for the out of plane orientation of the SQUID magnetic field relative to the plane of the sample is narrower than the in plane coercive field (see Table 1). This demonstrates an overall magnetic anisotropy of KHN-FePt nanocomposite films, originating from preferential distributions of FePt crystallites that control via magnetocrystalline anisotropy the coercivity of these 


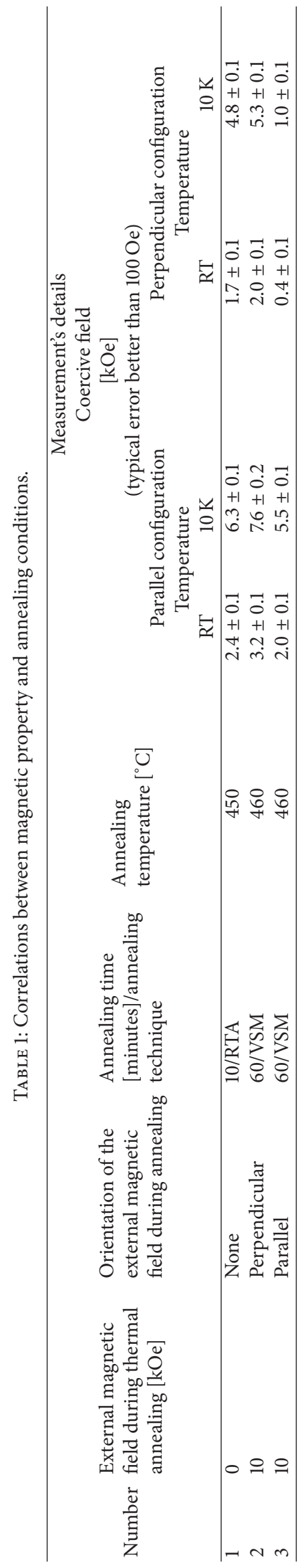



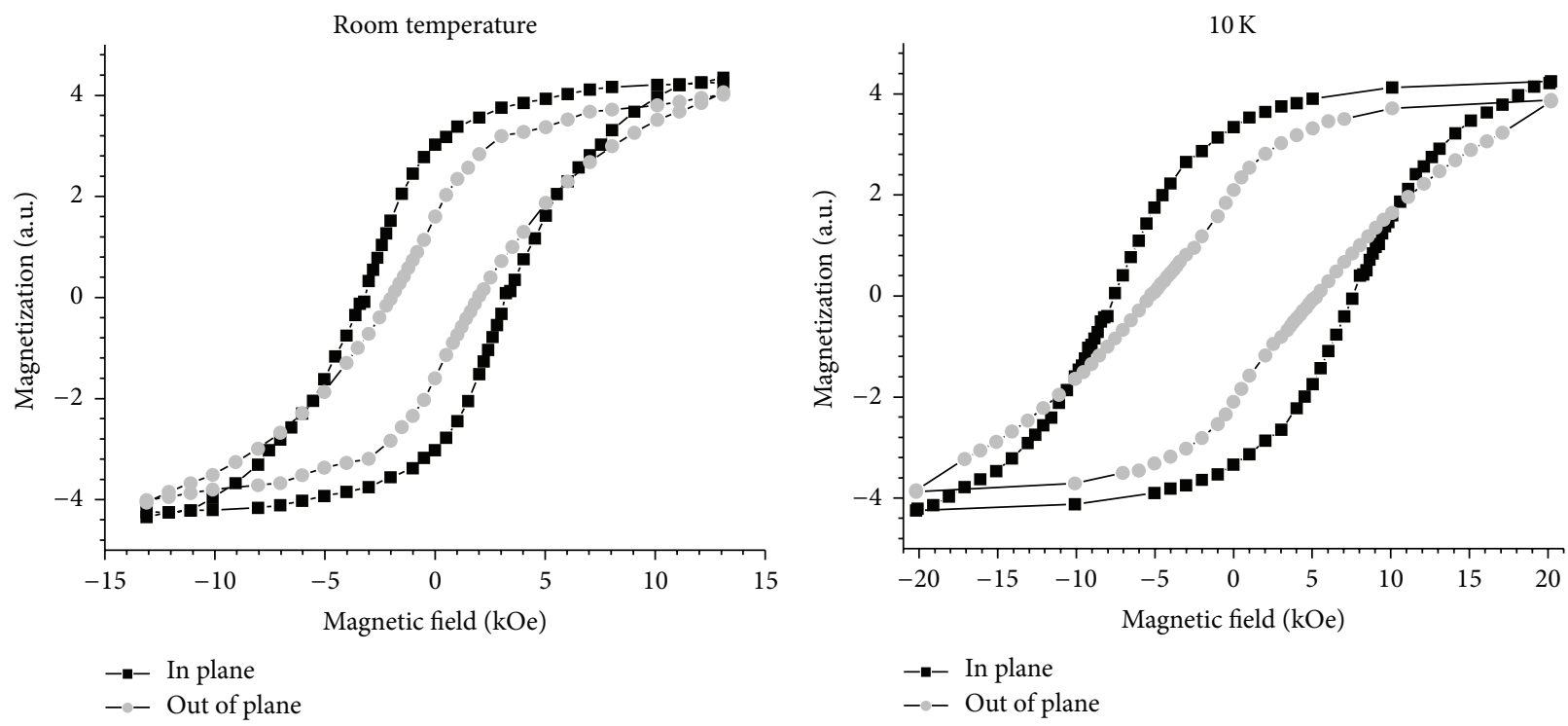

(a)
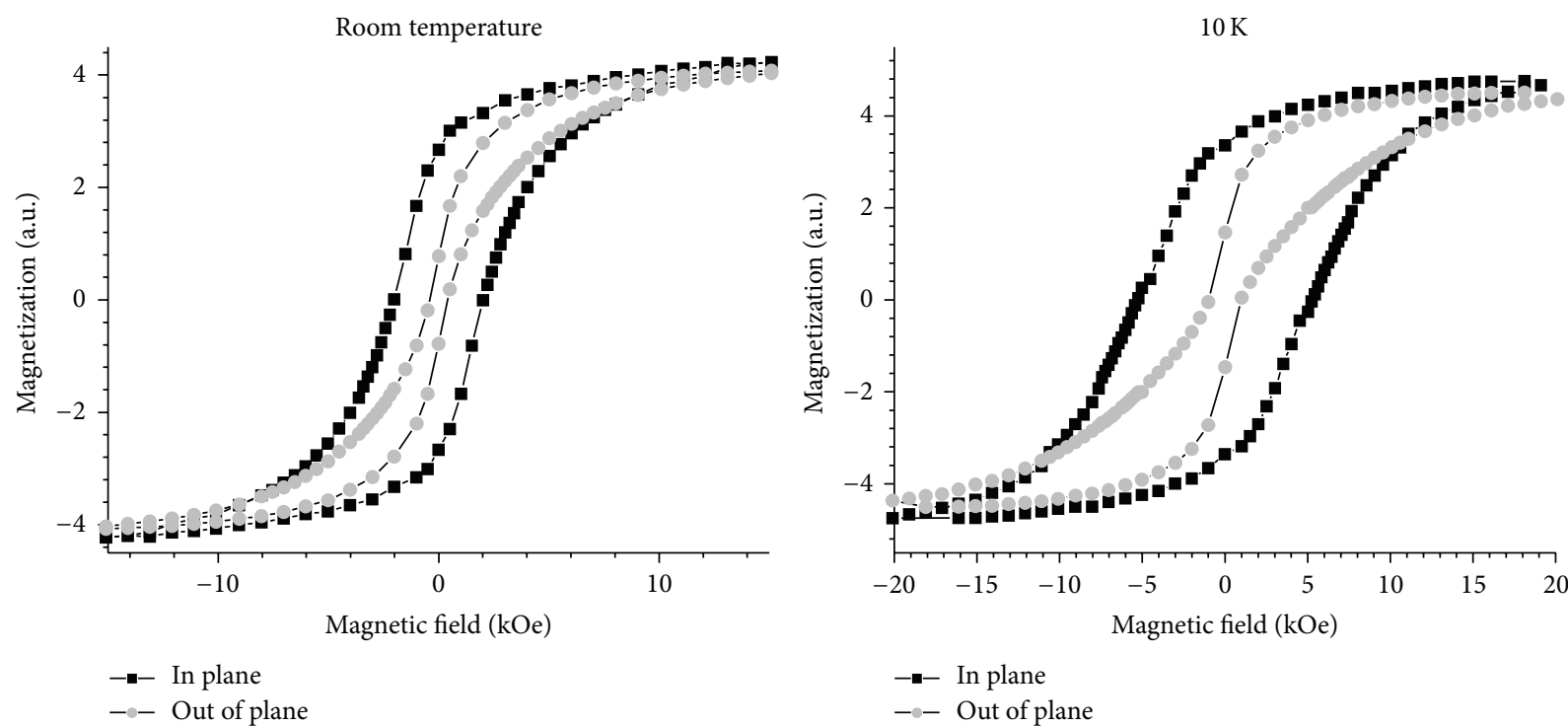

(b)

Figure 4: Hysteresis loops of KHN-FePt nanocomposite annealed at $460^{\circ} \mathrm{C}$ for $1 \mathrm{~h}$, in an external magnetic field of 10,000 Oe. (a) Samples annealed in magnetic field perpendicular to the plane of the sample. (b) Samples annealed in magnetic field parallel to the plane of the sample.

samples. As expected, the coercivity is significantly increased as the temperature is decreased to $10 \mathrm{~K}$.

The electron spin resonance (ESR) spectrum of FePt nanoclusters deposited onto Kapton, shown in Figures 5(a) and 5(b), is a convolution of two lines: a faint and narrow line due to the local degradation of the polymer matrix and assigned to graphite-like structures (describing the so-called electron paramagnetic mode of the ESR spectrometer) and a strong and broad line that exhibits angular dependence assigned to electronic spins coupled by magnetic interactions (reflecting the ferromagnetic resonance mode of the ESR spectrometer). The narrow line was observed in all samples bombarded by $\mathrm{FePt}$ (both annealed and not annealed) while pristine polyimide showed no ESR signal. Analogous spectra were reported in ion-beam-irradiated polymers (including polyimide), where they were assigned to graphite-like structures created by localized heating of the polymer during ion bombardment [34].

The shift of the of the ferromagnetic resonance (FMR) line position from the characteristic value corresponding to $g=2.00$ (see Figure 5(b)) reflects the effect of an additional average local field acting on the electronic spins. The angular dependence of the FMR signal observed for all samples (shown in Figure 5(b) for the not annealed sample) demonstrates that the additional field is likely a consequence of magnetocrystalline anisotropy (and may include 


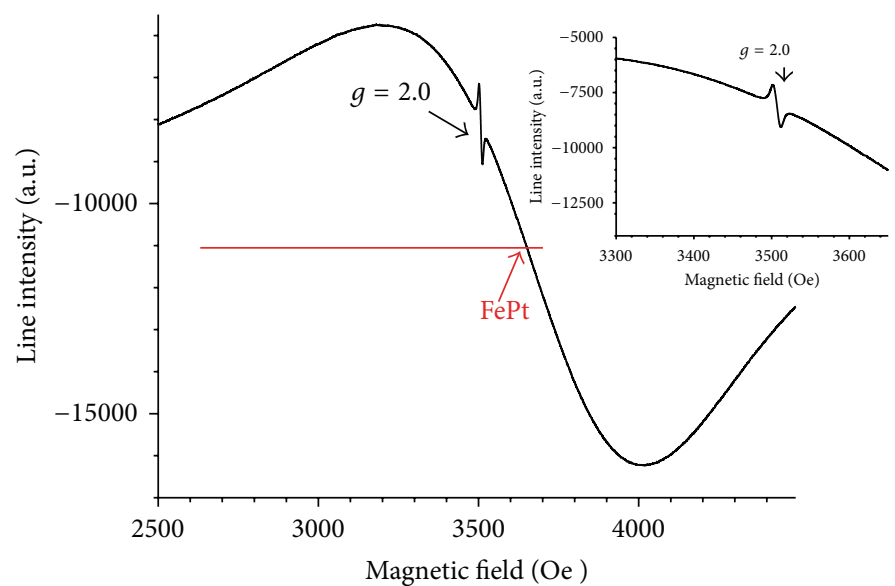

(a)

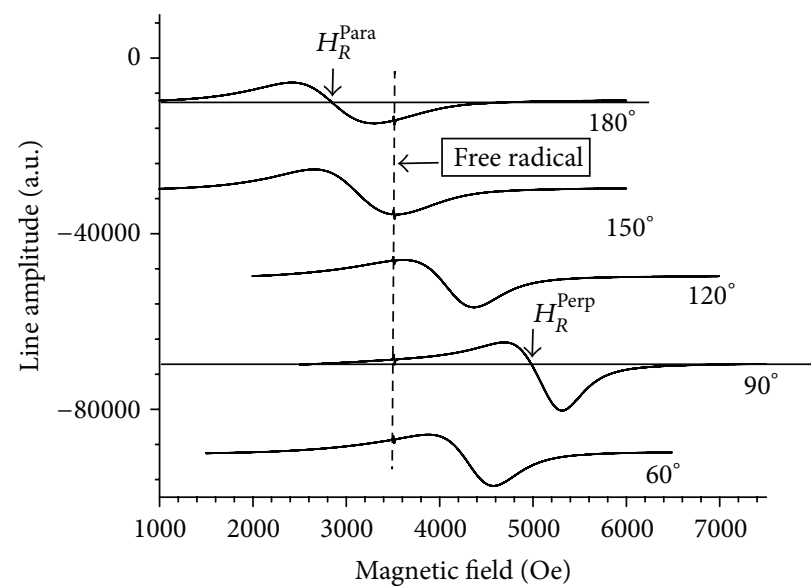

(b)

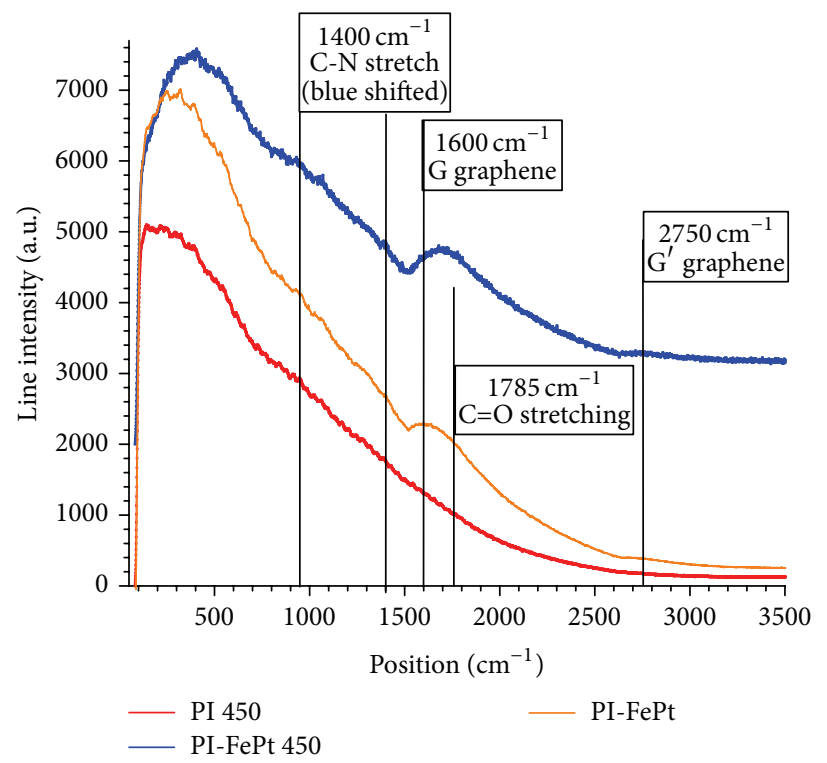

(c)

Figure 5: (a) ESR spectrum of not annealed KHN-FePt. (b) Angular dependence of ESR spectrum (not annealed KHN-FePt). (c) Raman spectra of annealed KHN, as-deposited KHN-FePt and annealed KHN-FePt.

magnetoelastic effects and even very small shape anisotropy contributions). The positions of the resonance fields $H_{R}^{\text {Para }}$ and $H_{R}^{\text {Perp }}$ for the parallel and perpendicular configurations are [35]

$$
\begin{aligned}
\frac{\hbar \omega}{g \mu_{B}} & =H_{R}^{\text {Perp }}-4 \pi M_{\mathrm{eff}}, \\
\left(\frac{\hbar \omega}{g \mu_{B}}\right)^{2} & =H_{R}^{\text {Para }}\left(H_{R}^{\text {Para }}+4 \pi M_{\mathrm{eff}}\right),
\end{aligned}
$$

where $M_{\text {eff }}=M_{S}-H_{K} /(4 \pi), \hbar$ is Planck's constant, $g$ is the gyromagnetic factor (about 2.0036), $\mu_{B}$ is the Bohr magneton, and $M_{\text {eff }}$ is the effective magnetization, $M_{S}$ is the magnetization at saturation, and $K_{1}$ represents the effective perpendicular anisotropy field [35]. The effective anisotropy magnetization and field can be estimated from the position of the resonance fields in the parallel and perpendicular configurations [35]:

$$
\begin{aligned}
4 \pi M_{\mathrm{eff}}= & H_{R}^{\text {Perp }}+\left(\frac{H_{R}^{\text {Para }}}{2}\right) \\
& -\left[H_{R}^{\text {Para }}\left(H_{R}^{\text {Perp }}+\left(\frac{5}{4}\right) H_{R}^{\text {Para }}\right)\right]^{1 / 2}=H_{\text {eff }}
\end{aligned}
$$

From Figure 5(b) it is determined that $H_{R}^{\text {Para }}=2850 \pm 5$ Oe, and $H_{R}^{\text {Perp }}=4980 \pm 5$ Oe resulting in an effective anisotropy field, $H_{\text {eff }}$, of about $6300 \pm 5$ Oe for the not annealed sample, at room temperature. This result agrees to the observed anisotropic hysteresis loops. Actually, both the position and width of the ferromagnetic resonance line of both annealed and not annealed samples were dependent on the orientation of the sample with respect to the static external field of the ESR spectrometer. 
Raman spectroscopy (see Figure 5(c)) supports the formation of graphitic structures in bombarded and thermal annealed KHN. The broad lines observed at 1800 and $2750 \mathrm{~cm}^{-1}$ and assigned to the $\mathrm{G}$ and, respectively, $\mathrm{G}^{\prime}$ modes are supporting the formation of graphitic structures [36]. However, the Raman spectra of KHN bombarded by FePt and/or annealed are controlled by broad components due to strong luminescence contributions derived from the presence of carbonaceous structures [36].

\section{Conclusions}

This report focuses on a research of FePt nanoclusters implanted within a flexible polymeric film. Due to the local energy deposited within the polymeric target during the implantation, a local carbonization process occurring within the polymeric substrate is expected. The actual shape of FePt nanoclusters may include a weak distortion from a spherical towards an ellipsoidal shape with the long axis normal to the surface of the substrate. This may contribute to the magnetic anisotropy of the as-deposited samples. The local degradation of bombarded polymeric films has been reported in ion beam irradiated polymers [34] including polyimide $[37,38]$. Consequently, the system consisting of FePt clusters implanted within KHN should have features analogous to the FePt:C system (where the FePt clusters are expected to be coated by a very this carbon layer, generated by the destruction of the macromolecular chain [37]).

FePt nanoclusters with an average diameter of $5 \mathrm{~nm}$ have been implanted within Kapton. The implantation resulted in nanoclusters of FePt coated with a graphite-like material due to the heat released during the stopping of clusters within the polymeric film. However, as the implantation process occurs at temperatures significantly below the melting temperature of FePt or the decomposition temperature of KHN, isolated quasispherical FePt nanoparticles are expected within the polymeric substrate. This is in agreement with the observed SEM micrographs (on annealed samples). The annealing temperatures were selected below the melting/decomposition temperature of the polymer and of FePt (at all annealing temperature both the polymer and the nanoparticles were solid). Consequently, no coalescence/agglomeration process was noticed. Even more, the width of the WAXS line was not affected by annealing suggesting that the size of FePt crystallites did not change significantly during the annealing step. The as-obtained system mimicked a dilute FePt:C [12] system dispersed within an elastic polymeric matrix. However, during the implantation of FePt clusters the surface of the polyimide film was wrinkled. This might be avoided by increasing the temperature of the polyimide film during the bombardment with FePt clusters. The temperature dependence of the magnetic properties of annealed and pristine polyimide-FePt films in the temperature range $10 \mathrm{~K}$ to $750 \mathrm{~K}$ has been investigated. The blocking temperature for the not annealed film was estimated to about $70 \mathrm{~K}$ and shifted above room temperature after thermal annealing, because of the ordering of $\mathrm{FePt}$ and of a large increase in anisotropy constant. Based on WAXS data it was concluded that the pristine KHN-FePt system contained crystalline FePt, with a low content of $\mathrm{Ll}_{0}$ phase. Thermal annealing confirmed the enhancement of the $\mathrm{Ll}_{0}$ phase but the stability of the polymeric substrates prevented high temperature annealing (above $550^{\circ} \mathrm{C}$ ).

This study has demonstrated the possibility to produce flexible magnetic films by bombarding free standing polymeric films with FePt nanoclusters and obtaining the $\mathrm{L}_{0}$ phase by thermal annealing. It also revealed film degradation generated by the local heating of the polymeric matrix. The thermal annealing in external magnetic fields further improved the orientation of FePt nanoclusters within the polymeric film.

\section{Conflict of Interests}

The authors declare that there is no conflict of interests regarding the publication of this paper.

\section{Acknowledgment}

This research was funded by Faculty Research Council (UTPA) and NSF-MRSEC (Nebraska-Lincoln).

\section{References}

[1] Y. F. Xu, M. L. Yan, and D. J. Sellmyer, "Cluster-assembled nanocomposites," in Advanced Magnetic Nanostructures, D. J. Sellmyer and R. Skomski, Eds., pp. 207-238, Springer, New York, NY, USA, 2006.

[2] X. Rui, Z. G. Sun, Y. Xu, D. J. Sellmyer, and J. E. Shield, “Clusterassembled exchange-spring nanocomposite permanent magnets," Journal of Applied Physics, vol. 97, no. 10, Article ID 10K310, 2005.

[3] D. E. Laughlin, K. Srinivasan, M. Tanase, and L. Wang, "Crystallographic aspects of $\mathrm{L}_{0}$ magnetic materials," Scripta Materialia, vol. 53, no. 4, pp. 383-388, 2005.

[4] S. Anders, M. F. Toney, T. Thomson et al., "X-ray absorption and diffraction studies of thin polymer/FePt nanoparticle assemblies," Journal of Applied Physics, vol. 93, no. 10, pp. 6299-6304, 2003.

[5] A. C. Sun, F. T. Yuan, and J. Hsu, "Control of growth and ordering process in $\mathrm{FePt}(001)$ film at $300^{\circ} \mathrm{C}$," Journal of Physics: Conference Series, vol. 200, no. 10, Article ID 102009, 2010.

[6] S. D. Granz and M. H. Kryder, "Granular $\mathrm{L1}_{0}$ FePt (001) thin films for Heat Assisted Magnetic Recording," Journal of Magnetism and Magnetic Materials, vol. 324, no. 3, pp. 287-294, 2012.

[7] K. M. Hyie and I. I. Yaacob, "Annealing behavior of ferromagnetic FePt nanoparticles prepared in water in oil microemulsions," in Proceedings of the World Congress on Engineering (WCE '08), vol. 2, pp. 2-5, London, UK, July 2008.

[8] Y. F. Ding, J. S. Chen, E. Liu, B. C. Lim, J. F. Hu, and B. Liu, "Effect of carbon additive on microstructure evolution and magnetic properties of epitaxial FePt (001) thin films," Thin Solid Films, vol. 517, no. 8, pp. 2638-2647, 2009.

[9] M. Mizuguchi, T. Sakurada, T. Y. Tashiro, K. Sato, T. J. Konno, and K. Takanashi, "Fabrication of highly $\mathrm{L1}_{0}$-ordered $\mathrm{FePt}$ thin films by low-temperature rapid thermal annealing," $A P L$ Materials, vol. 1, no. 3, Article ID 032117, 2013. 
[10] R. Skomski, A. Kashyap, and J. Zhou, "Atomic and micromagnetic aspects of $\mathrm{Ll}_{0}$ magnetism," Scripta Materialia, vol. 53, no. 4, pp. 389-394, 2005.

[11] M. Chipara, D. Hui, J. Sankar et al., "On styrene-butadienestyrene-barium ferrite nanocomposites," Composites Part B: Engineering, vol. 35, no. 3, pp. 235-243, 2004.

[12] Y. Xu, M. L. Yan, J. Zhou, and D. J. Sellmyer, "Magnetic properties of dilute FePt:C nanocluster films," Journal of Applied Physics, vol. 97, no. 10, Article ID 10J320, 2005.

[13] N. H. Nam, N. T. T. Van, N. D. Phu, T. T. Hong, N. Hoang Hai, and N. H. Luong, "Magnetic properties of FePt nanoparticles prepared by sonoelectrodeposition," Journal of Nanomaterials, vol. 2012, Article ID 801240, 4 pages, 2012.

[14] M. Yu, H. Ohguchi, A. Zambano et al., "Orientation and magnetic properties of FePt and CoPt films grown on $\mathrm{MgO}(1$ $10)$ single-crystal substrate by electron-beam coevaporation," Materials Science and Engineering B, vol. 142, no. 2-3, pp. 139143, 2007.

[15] J. Fang, K. Stokes, J. He, W. L. Zhou, and J. C. O’Connor, “Synthesis and magnetic properties of CoPt-poly(methylmethacrylate) nanostructured composite material," Journal of Applied Physics, vol. 91, no. 10, pp. 8816-8818, 2002.

[16] V. Salgueiriño-Maceira, M. A. Correa-Duarte, E. Duman, and M. Farle, "FePt nanocrystals embedded in methylmethacrylate polymers," Journal of Magnetism and Magnetic Materials, vol. 299, no. 2, pp. 467-471, 2006.

[17] H. M. Song, Y. J. Kim, and J. H. Park, "Three-dimensional hierarchically organized magnetic nanoparticle polymer composites: achievement of monodispersity and enhanced tensile strength," The Journal of Physical Chemistry C, vol. 112, no. 14, pp. 5397-5404, 2008.

[18] H. Wakayama, H. Yonekura, and Y. Kawai, “Three-dimensional periodically ordered nanohetero metallic materials from selfassembled block copolymer composites," ACS Macro Letters, vol. 2, no. 4, pp. 284-287, 2013.

[19] T. Song, Y. Z. Zhang, and T. J. Zhou, "Fabrication of magnetic composite nanofibers of poly( $\varepsilon$-caprolactone) with $\mathrm{FePt}$ nanoparticles by coaxial electrospinning," Journal of Magnetism and Magnetic Materials, vol. 303, no. 2, pp. e286-e289, 2006.

[20] T. Song, Y. Zhang, T. Zhou, C. T. Lim, S. Ramakrishna, and B. Liu, "Encapsulation of self-assembled FePt magnetic nanoparticles in PCL nanofibers by coaxial electrospinning," Chemical Physics Letters, vol. 415, no. 4-6, pp. 317-322, 2005.

[21] M. Wang, H. Singh, T. A. Hatton, and G. C. Rutledge, "Fieldresponsive superparamagnetic composite nanofibers by electrospinning," Polymer, vol. 45, no. 16, pp. 5505-5514, 2004.

[22] Y. Tamada, S. Yamamoto, S. Nasu, and T. Ono, "Structural and magnetic properties of $\mathrm{Ll}_{0}$-FePt nanoparticles aligned by external magnetic field," Physical Review B-Condensed Matter and Materials Physics, vol. 78, no. 21, Article ID 214428, 2008.

[23] H. S. Virk, "Physical and chemical response of $70 \mathrm{MeV}$ carbon ion irradiated Kapton-H polymer," Nuclear Instruments and Methods in Physics Research, Section B: Beam Interactions with Materials and Atoms, vol. 191, no. 1-4, pp. 739-743, 2002.

[24] G.-M. Choi, B.-C. Min, and K.-H. Shin, "L1 $1_{0}$ ordering of FePtB films on a thin MgO layer," Applied Physics Express, vol. 4, no. 2, Article ID 023001, 2011.

[25] T. J. Klemmer, N. Shukla, C. Liu et al., "Structural studies of $L 1_{0}$ FePt nanoparticles," Applied Physics Letters, vol. 81, no. 12, article 2220, 2002.
[26] K. E. Elkins, T. S. Vedantam, J. P. Liu et al., "Ultrafine FePt nanoparticles prepared by the chemical reduction method," Nano Letters, vol. 3, no. 12, pp. 1647-1649, 2003.

[27] B. H. Sohn, R. E. Cohen, and G. C. Papaefthymiou, "Magnetic properties of iron oxide nanoclusters within microdomains of block copolymers," Journal of Magnetism and Magnetic Materials, vol. 182, no. 1-2, pp. 216-224, 1998.

[28] G. S. Rajan, S. L. Stromeyer, K. A. Mauritz et al., "Superparamagnetic nanocomposites based on poly(styrene- $b$-ethylene/butylene-b-styrene)/cobalt ferrite compositions," Journal of Magnetism and Magnetic Materials, vol. 299, no. 1, pp. 211-218, 2006.

[29] Y. Xu, M. L. Yan, and D. J. Sellmyer, "Cluster-assembled nanocomposites," in Advanced Magnetic Nanostructures, pp. 207-238, Springer, 2006.

[30] S. Okamoto, N. Kikuchi, O. Kitakami, and Y. Shimada, "Magnetization reversal process in $\mathrm{FePt} \mathrm{Ll}_{0}$ nanoparticles," Scripta Materialia, vol. 53, no. 4, pp. 395-401, 2005.

[31] Y. K. Takahashi, K. Hono, S. Okamoto, and O. Kitakami, "Magnetization reversal of FePt hard/soft stacked nanocomposite particle assembly," Journal of Applied Physics, vol. 100, no. 7, Article ID 074305, 2006.

[32] N. Kikuchi, K. Mitsuzuka, T. Shimatsu, O. Kitakami, and H. Aoi, "Nucleation size of hcp-CoPt dot arrays characterized by time dependence of coercivity," Journal of Physics: Conference Series, vol. 200, no. 10, Article ID 102003, 2010.

[33] T. Shimatsu, H. Uwazumi, H. Muraoka, and Y. Nakamura, "Thermal stability in perpendicular recording media," Journal of Magnetism and Magnetic Materials, vol. 235, no. 1-3, pp. 273280, 2001.

[34] M. I. Chipara, I. Bunget, R. Georgescu, E. Georgescu, and I. Vilcov, "ESR studies on pet irradiated with high energy ions," Nuclear Instruments and Methods in Physics Research, vol. 209210, no. 1, pp. 395-400, 1982.

[35] A. Martins, S. C. Trippe, A. D. Santos, and F. Pelegrini, "Spinwave resonance and magnetic anisotropy in FePt thin films," Journal of Magnetism and Magnetic Materials, vol. 308, no. 1, pp. 120-125, 2007.

[36] D. M. Chipara, A. C. Chipara, and M. Chipara, "Raman spectroscopy of carbonaceous materials: a concise review," Spectroscopy, vol. 26, no. 10, pp. 2-7, 2011.

[37] J.-M. Costantini, J.-P. Salvetat, F. Couvreur, and S. Bouffard, "Carbonization of polyimide by swift heavy ion irradiations: effects of stopping power and velocity," Nuclear Instruments and Methods in Physics Research, Section B: Beam Interactions with Materials and Atoms, vol. 234, no. 4, pp. 458-466, 2005.

[38] D. Fink, R. Klett, L. Chadderton et al., "Carbonaceous clusters in irradiated polymers as revealed by small angle X-ray scattering and ESR," Nuclear Instruments and Methods in Physics Research Section B: Beam Interactions with Materials and Atoms, vol. 111, no. 3-4, pp. 303-314, 1996. 

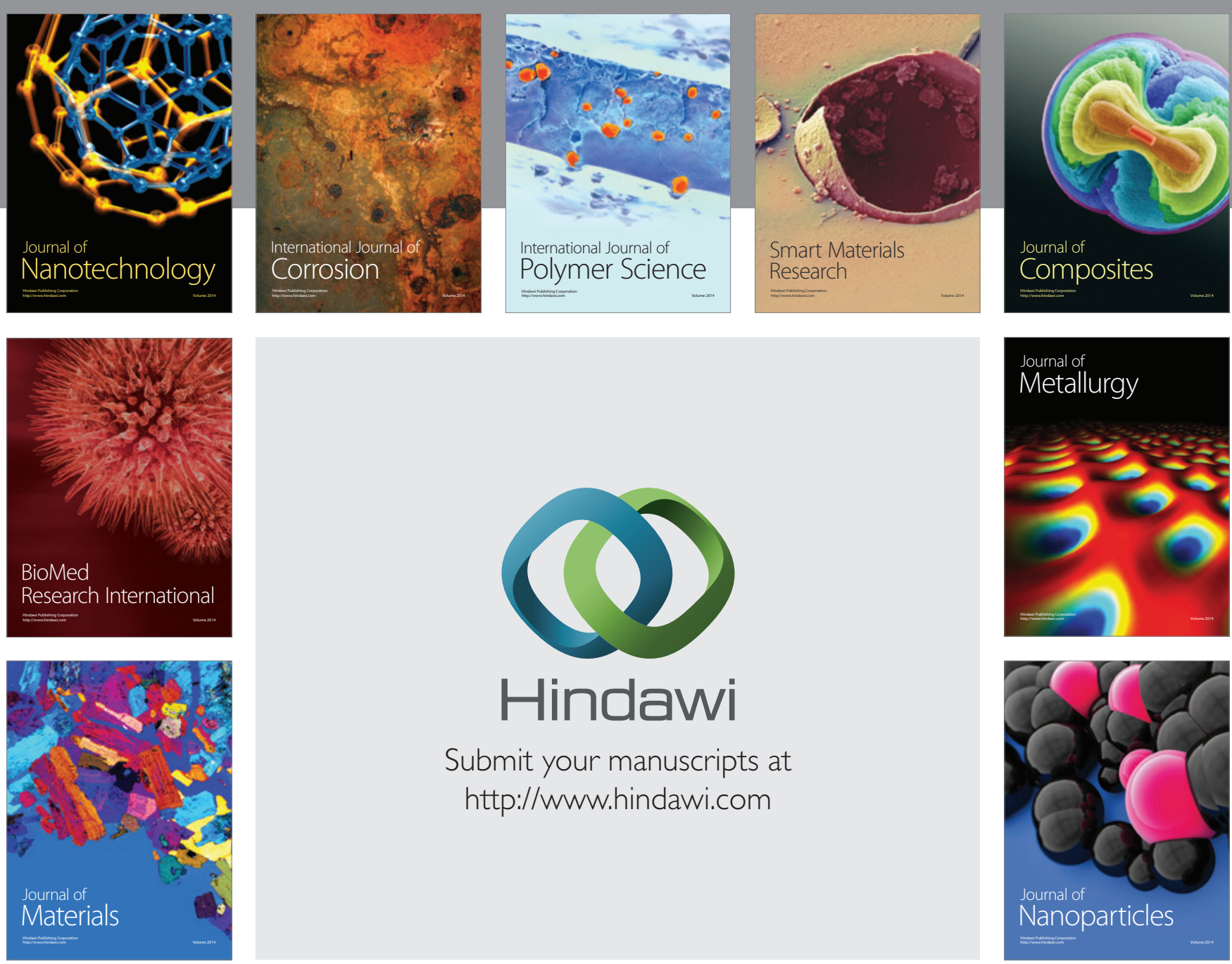

Submit your manuscripts at http://www.hindawi.com
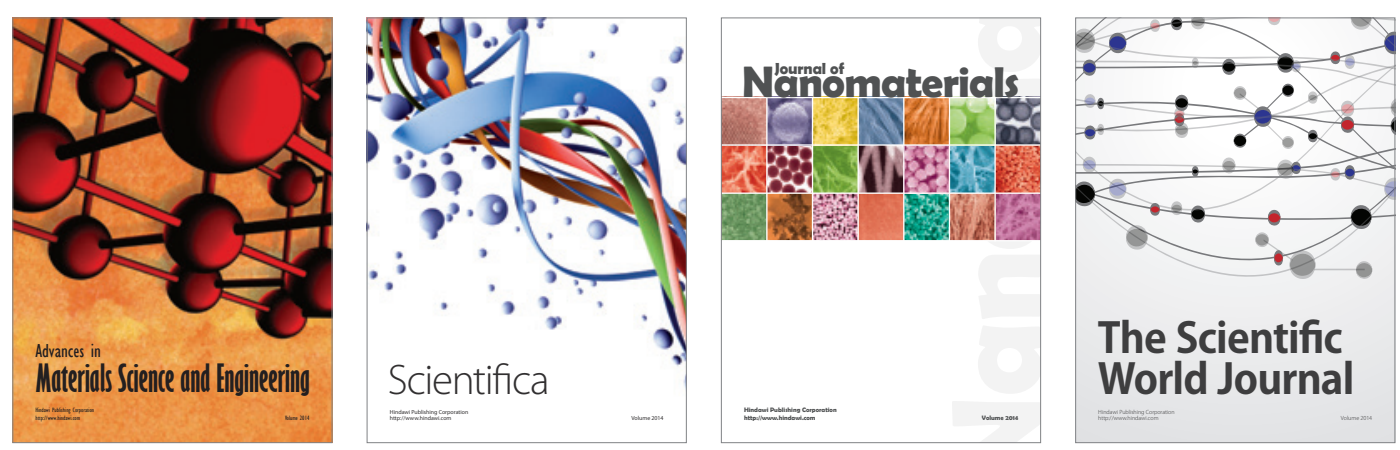

\section{The Scientific World Journal}
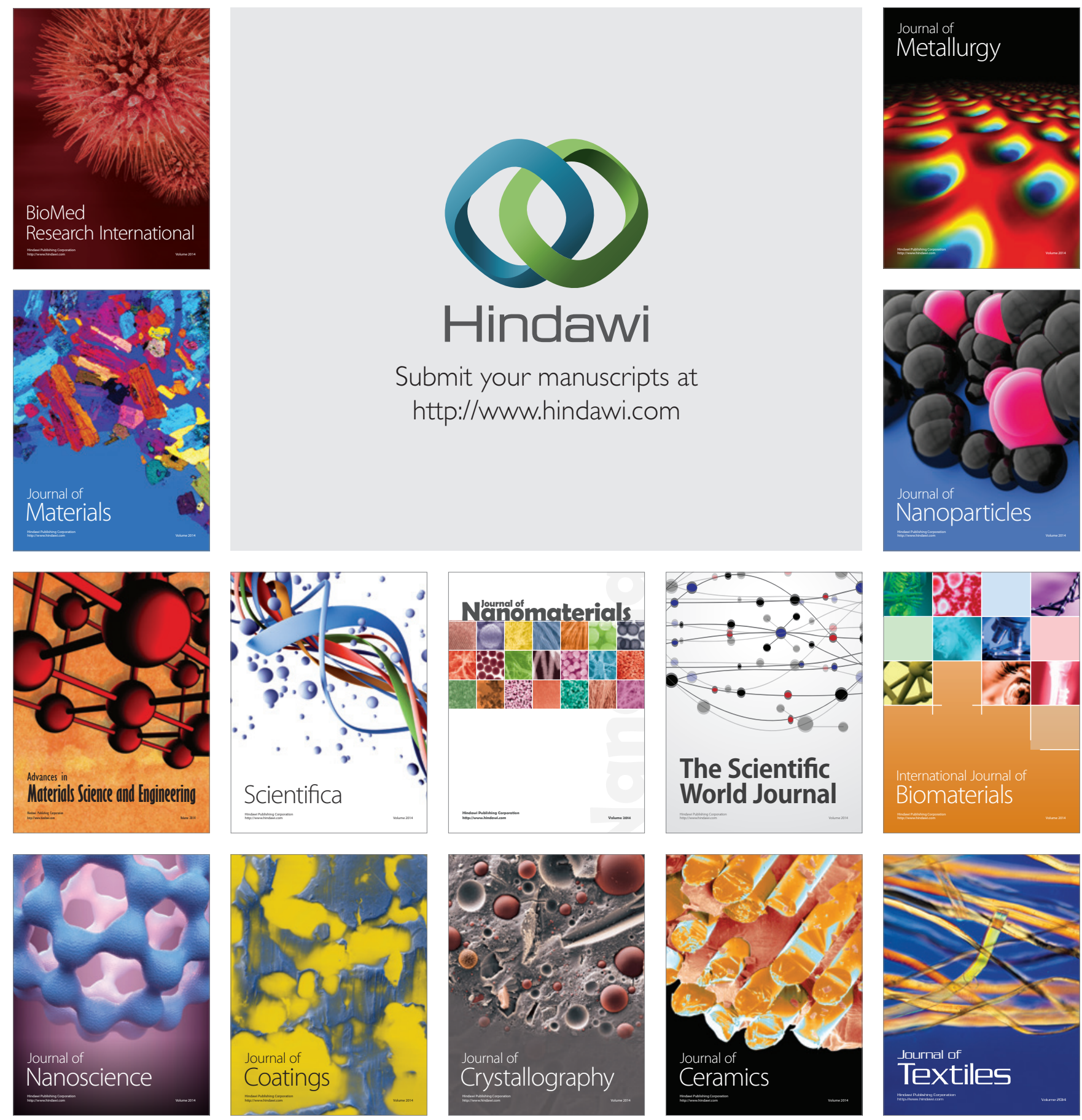